\section{UNA EVALUACIÓN ON-LINE A LA DEMANDA PARA EL AUTOAPRENDIZAJE}

\author{
Miguel Balbás \\ Agustín García-Berrocal \\ Cristina Montalvo \\ J. Ignacio Díaz de Villafranca \\ Departamento de Física Aplicada a los Recursos Naturales, \\ ETSI de MINAS, UPM, C/Ríos Rosas 21, 28003 Madrid \\ miguel.balbas@upm.es
}

\section{ON LINE EVALUATION ON DEMAND FOR SELF-LEARNING}

ABSTRACT: In the School of Mines of the Technical University of Madrid (UPM) the adaptation process to the Bologna principles have been carried out. This has implied changes in the teaching methodology. In this paper a new methodology is described and it is applied to an introductory course of basic mechanics of the first semester of two different engineering degrees. The results are presented through an index which permits to assess the global results of the teachinglearning process reached with this methodology.

KEY WORDS: Self-learning: self-evaluation; continuous evaluation; on-line evaluation.

\section{INTRODUCCIÓN}

Las Universidades españolas están realizando el proceso de adaptación de sus enseñanzas al Espacio Europeo de Enseñanza Superior (EEES) (European Comission, 1999; Fernández, C. et al., 2011). Dentro de este proceso en la Escuela Técnica Superior de Ingenieros de Minas, de la Universidad Politécnica de Madrid, se ha desarrollado la experiencia metodológica que se describe, en la asignatura denominada Física I perteneciente al curso inicial en la formación de grado de Ingeniería. Los contenidos de la asignatura corresponden a un curso básico de Mecánica.

La adaptación exige un cambio metodológico que facilite una profundización en las ideas-fuerza. El proceso de enseñanza-aprendizaje viene establecido por los objetivos pedagógicos, el perfil de los estudiantes, la metodología elegida y el método de evaluación que se aplique. El alumnado, por un lado, es muy joven, como corresponde a su llegada a la universidad, y por otro presenta un nivel
RESUMEN: En la Escuela de Minas de la Universidad Politécnica de Madrid (UPM) se ha llevado a cabo el proceso de adaptación a los principios de Bolonia. Esto ha implicado cambios en la metodología de la enseñanza. En este artículo se describe una nueva metodología aplicada a un curso introductorio de Mecánica ubicado en el primer semestre del grado de dos diferentes títulos de ingeniería. Los diferentes resultados se presentan mediante un indice que permite evaluar el resultado global del proceso de enseñanza-aprendizaje alcanzado con esta metodología.

PALABRAS CLAVE: Autoaprendizaje; autoevaluación; evaluación continua; evaluación on-line.

alto de heterogeneidad en sus conocimientos científicos previos, estando además frecuentemente no demasiado motivado; es decir son alumnos con escasa homogeneidad en sus actitudes y en sus aptitudes.

Por otro lado, no se renuncia a alcanzar en el proceso de enseñanza-aprendizaje objetivos de niveles cognitivos medios y altos, como los de aplicación, análisis, evaluación y creación, según la taxonomía clásica y revisada de Bloom (Amer, A., 2006). Se ha optado, por ello, por una metodología que establezca claramente pautas para un trabajo personalizado, con autoaprendizaje y autoevaluación, de forma que el alumno pueda evaluar el nivel de logro de su aprendizaje en cada objetivo trazado, pudiendo decidir personalmente realizar un refuerzo de su aprendizaje hasta alcanzar un nivel suficiente.

El proceso va acompañado de un sistema de evaluación continua. Cada semana se debe alcanzar un objetivo, siguiendo la programación establecida. El nivel de logro 
de su alcance por parte del alumno, queda registrado, con incidencia en su calificación final, lo que debe servir de incentivo para mantener de forma continuada el esfuerzo del aprendizaje. Se ha establecido para los alumnos que han elegido seguir la evaluación continua, que la calificación final se logra sumando las calificaciones obtenidas en la evaluación continua y en el examen final. Tanto la evaluación continua como el examen pueden alcanzar una calificación de 5 puntos cada uno. Existe una condición restrictiva que obliga, para superar la asignatura, a alcanzar 1,5 puntos en el examen final (equivalente a 3 sobre 10). La evaluación continua a su vez tiene cinco componentes, alguna de las cuales trataremos más adelante.

En el proceso global de evaluación de la asignatura se consideran diferentes aspectos, tales como la comprensión, aplicación y análisis de los fundamentos teórico-prácticos, la resolución de problemas y los trabajos prácticos de laboratorio. La asignatura es de carácter semestral con una duración de 15 semanas. Los alumnos que han seguido esta metodología pertenecen a dos titulaciones: Graduados en Ingeniería de la Energía y en Ingeniería en Tecnología Minera. El número total de alumnos ha sido de 340 , todos ellos sin experiencia previa universitaria.

\section{Herramientas para el autoaprendizaje Y LA AUTOEVALUACIÓN}

El número de alumnos y la frecuencia de la evaluación son razones suficientes para elegir un soporte online, que por otro lado es más motivador para el alumno y le permite flexibilizar los tiempos de dedicación al aprendizaje (Kurt, A. et al., 2006; Neri, L. et al., 2010, y Stefanovic et al., 2010). En nuestra Universidad se utiliza la plataforma informática Moodle, que permite una gran variedad de aplicaciones. Cada alumno posee una clave personal que le permite en todo momento acceder al uso de la plataforma.

El desarrollo del programa de la asignatura comprende 15 temas, de duración semanal, clasificados en 6 temas de cinemática (cinemática del punto, cinemática de los sistemas y del sólido rígido, y cinemática del movimiento relativo a dos observadores en movimiento), 7 temas de dinámica (dinámica de la partícula, teoremas generales, energías cinética, potencial y mecánica, y dinámica de los sistemas y del movimiento plano del sólido) y 2 temas de estática (estática del sólido rígido plano). Cada uno de los temas tiene un objetivo específico y un logro de aprendizaje definido.

El alumno dispone de cuestionarios compuestos de varias preguntas que debe contestar, según el tema que elija. En la plataforma informática se ha creado una base de preguntas, de entre las cuales la plataforma elige al azar las que forman un cuestionario que se presenta en pantalla, siempre dentro del tema elegido. Las preguntas no son de respuesta inmediata, de solo conocimiento, sino que exigen para su respuesta un proceso de razonamiento. Después del enunciado de la cuestión, la plataforma presenta una cierta afirmación. El alumno debe identificar esta aseveración como correcta o incorrecta. Inmediatamente después de contestar, se le muestra en pantalla, haya acertado o no en su contestación, la respuesta correctamente razonada, preparada por sus profesores, y que él deberá comparar con la contestación que elaboró. En algunas ocasiones la respuesta incluye enlaces a explicaciones más detalladas, que el alumno puede consultar si lo considera oportuno. En algunas ocasiones la respuesta exige la realización de un cálculo auxiliar. El número de cuestiones contenidas en la base es de 600 .

La base de preguntas es accesible durante todo el curso para que el alumno pueda repasar o profundizar en los temas ya desarrollados o incluso ultimar su preparación del examen final. El alumno va comprobando la calificación que ha obtenido en cada logro de aprendizaje. Y el resultado de esta autoevaluación le ayudará a decidir si debe o no emplear más esfuerzo en cada uno de dichos logros. No obstante, la plataforma concede un tiempo de dos semanas para cada tema, la semana en que se desarrolla en clase el tema y la semana posterior, en el cual se registra la calificación del alumno en el tema. Siempre se conserva la calificación máxima que haya obtenido el alumno en las cuestiones contestadas, con el objetivo de no cortar la posibilidad de realizar más cuestionarios y proseguir el autoaprendizaje, por temor a obtener calificaciones menores en sucesivos intentos. Pasado este plazo sigue abierta la posibilidad de que el alumno vuelva sobre cuestionarios del mismo tema, pero ya no se tendrán en cuenta para valorar su logro en la componente de la evaluación continua por cuestionarios teórico-prácticos. 
Un cilindro puede realizar uno de estos dos movimientos: o bien girar en torno a su eje

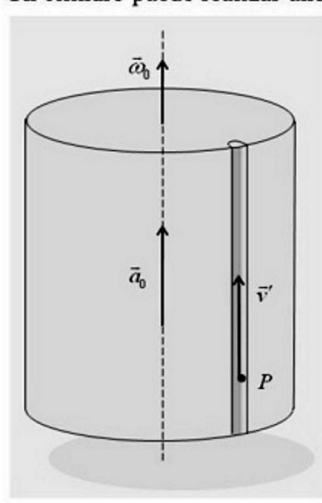
con velocidad angular $\bar{\omega}_{0}$ constante, o bien trasladarse con aceleración constante $\vec{a}_{0}$ en la dirección de su eje. No sabemos qué movimiento es el que se está realizando. Se sabe que un punto móvil recorre una ranura paralela al eje con velocidad relativa $\vec{v}^{\prime}$ de módulo constante.

Se puede afirmar que si nos dan el valor de la aceleración de Coriolis de $P$, puede deducirse que movimiento está haciendo el cilindro.
Un bloque, de peso $P_{1}$, descansa en equilibrio apoyándose sobre un segundo bloque, de peso $P_{2}$, que a su vez se apoya sobre un plano inclinado de ángulo $\varphi$ con la horizontal. El bloque superior está unido a la pared mediante un hilo paralelo al plano. Una de las caras del bloque inferior, paralelas al plano inclinado, es lisa y la otra tiene rozamiento.

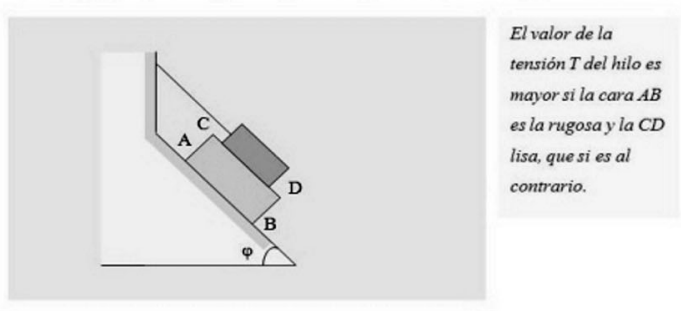

b)

Figura 1. Ejemplos de preguntas de Cinemática (a) y Estática (b)

Esta metodología tiene la ventaja, para el profesor, de que no requiere un trabajo adicional de corrección, ya que ésta es automática. Se incluyen, a continuación, dos ejemplos de cuestiones, uno de cinemática y otro de estática (Fig.1).

En cada una de estas cuestiones, el alumno debe razonar hasta concluir si la proposición final en cada una, escrita con otro tipo de letra, es correcta o incorrecta.

Los alumnos que han elegido esta modalidad de evaluación, realizan además dos pruebas presenciales a lo largo del curso, en las que tienen que responder, por escrito y razonadamente a dos cuestiones en cada una de las pruebas. La primera prueba se realiza al terminar las semanas dedicadas a la cinemática y la segunda al acabar el desarrollo de la dinámica. Estas pruebas tienen por objetivo verificar el aprendizaje, estimular al alumno para que ultime su aprendizaje en los plazos programados y comprobar la capacidad de expresión escrita de los alumnos. Las preguntas a responder son de la propia base, con lo que el alumno no tiene que hacer nada distinto a lo que ha venido practicando en su proceso de autoaprendizaje y su probabilidad de conocer la pregunta, por haberle salido en uno de sus cuestionarios, crece a medida que el alumno se esfuerza por responder a más cuestionarios.

De análoga manera en el examen final, junto con los problemas a resolver, se le presenta a los alumnos cua- tro cuestiones a resolver, dos de ellas sin variación ninguna respecto a la redacción que tienen en la base, una con modificaciones en su redacción y una última de nueva creación. El peso global que se le atribuye a este conjunto de cuestiones es del $50 \%$ del total de la calificación. Nuevamente puede afirmarse que el trabajo continuo con los cuestionarios facilita la respuesta de esta parte del examen final. Por último a los alumnos con mejor calificación se les ofrece una posibilidad de optar a una calificación de excelencia. Para ello deben crear y redactar cinco cuestiones (dos de cinemática, dos de dinámica y una de estática) con sus correspondientes respuestas.

\section{Resultados}

Para evaluar la eficacia de la metodología implantada, se han clasificado los alumnos en tres grupos según el seguimiento que han hecho del trabajo semanal de respuesta a los cuestionarios. El primer grupo está formado por los alumnos que ha realizado un seguimiento completo, es decir, que han trabajado y contestado a cuestionarios de todos los temas del programa; su número es alto, alcanzando prácticamente la mitad de la población. El segundo grupo lo constituyen los alumnos que han trabajado con cuestionarios de todos los temas, menos con uno, dos o tres como máximo. Por último el tercer grupo lo forman 
los alumnos que han hecho un seguimiento medio o bajo. No se ha dividido este grupo en dos, porque el número de alumnos de cada grupo sería demasiado pequeño. En la tabla 1 se recoge la composición de los tres grupos descritos, su tasa de éxito, su calificación final media y la calificación media en los cuestionarios.

TABLA 1: GRUPOS DE DIFERENTES SEGUIMIENTOS

\begin{tabular}{|l|c|c|c|c|c|}
\hline Grupo Seguimiento & N. ${ }^{2}$ alumnos & Porcentaje (\%) & Tasa de éxito & $\begin{array}{c}\text { Calificación final } \\
\text { media }\end{array}$ & $\begin{array}{c}\text { Calificación } \\
\text { cuestionarios } \\
\text { evaluados }\end{array}$ \\
\hline Completo & 169 & 49,7 & $71,6 \%$ & 5,7 & 0,97 \\
\hline Alto & 92 & 27,1 & $57,6 \%$ & 4,5 & 0,82 \\
\hline Medio/Bajo & 79 & 23,2 & $11,4 \%$ & 1,5 & 0,26 \\
\hline
\end{tabular}

Era de esperar una correlación entre el grado de seguimiento realizado por los alumnos y la calificación obtenida en las dos pruebas presenciales que se han descrito, la primera al finalizar en clase la cinemática y la segunda al terminar la dinámica. Los resultados obtenidos, promediando ambas pruebas sobre una escala de 20 puntos máximos, se muestran en la tabla 2, al igual que la calificación en las cuestiones teorico-prácticas del examen final (sobre 40) y la calificación (sobre 40) de la pregunta teorico-práctica nueva del examen final (la única de las cuatro que no puede conocer ningún alumno previamente, por no estar en la base Moodle).

La tabla 5 refleja, además de la relación estudiada, un nivel alto de dificultad en esta parte del examen final. En el gráfico de la figura 4, la información contenida en las tablas 1 y 2 se resume visualmente de manera radial, habiendo normalizado todos los valores a una escala de 10 puntos.
El aprendizaje obtenido mediante el trabajo individual con los cuestionarios tiene influencia en varias componentes de la evaluación sumativa. Para sintetizar estos diferentes resultados mostrados en las tablas anteriores, se ha creado un cuaternión $q$ (número hipercomplejo) (Gurlebeck, K. et al., 1997) que describa el fruto del aprendizaje. Se le asigna como parte real del número la tasa de éxito, y como sus tres partes imaginarias las calificaciones en los cuestionarios, las calificaciones medias en las pruebas presenciales y las calificaciones medias en las cuestiones TP del examen final, todos los valores numéricos llevados a una escala de 10 puntos. Cada cuaternión tiene un módulo que refleja numéricamente la cantidad de aprendizaje en cada grupo. Lo tomaremos como índice I del resultado global. También tiene una fase, fácilmente calculable. El valor máximo posible del módulo es 20 y la fase óptima es $60^{\circ}$. Esta fase indica el equilibrio entre las diferentes componentes de la evaluación continua. Podría decirse que refleja la calidad del aprendizaje. Si alguna de estas

TABLA 2: RELACIÓN DEL SEGUIMIENTO CON LAS PRUEBAS PRESENCIALES. CALIFICACIÓN SOBRE 20 PUNTOS

\begin{tabular}{|l|c|c|c|}
\hline \multicolumn{1}{|c|}{ Grupo Seguimiento } & $\begin{array}{c}\text { Calificación de las pruebas } \\
\text { (sobre 20) }\end{array}$ & $\begin{array}{c}\text { Calificación de cuestiones TP } \\
\text { en el examen final (sobre 40) }\end{array}$ & $\begin{array}{c}\text { Calificación de la cuestión TP } \\
\text { nueva en el examen final } \\
\text { (sobre 40) }\end{array}$ \\
\hline Completo & 12,3 & 18,8 & 20,8 \\
\hline Alto & 10,6 & 14,6 & 15,5 \\
\hline Medio/Bajo & 3,1 & 4,3 & 5,0 \\
\hline
\end{tabular}




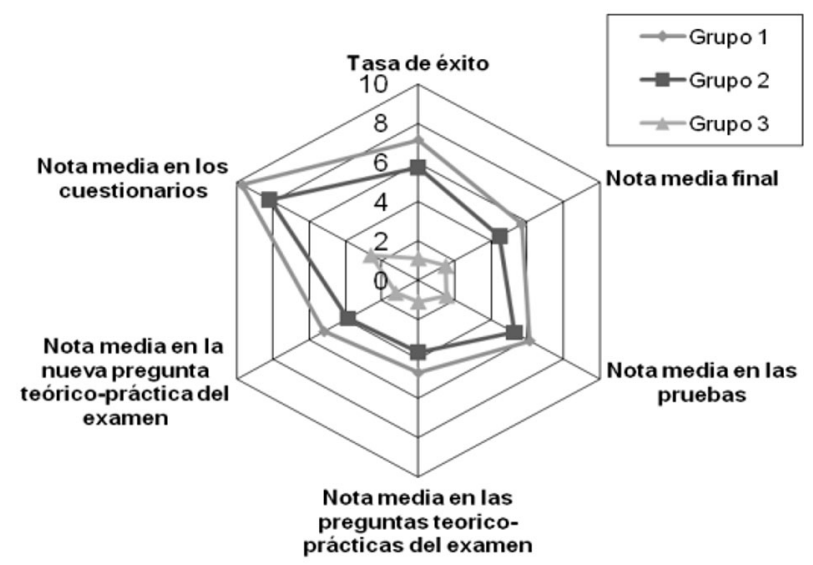

Figura 2. Carta de resultados de los tres grupos estudiados

componentes se desequilibra en el proceso, la fase acusa una diferencia con la fase óptima. Los valores obtenidos de los cuaterniones, así como sus módulos y fases de los tres grupos de seguimiento se muestran en la tabla 3.

TABLA 3: CUATERNIONES OBTENIDOS POR LOS DIFERENTES GRUPOS DE SEGUIMIENTO

\begin{tabular}{|l|c|c|c|}
\hline $\begin{array}{c}\text { Grupo de } \\
\text { Segumiento }\end{array}$ & Cuaternión q & Índice I & Equilibrio F \\
\hline Completo & $(7,16 ; 9,70,6,15,4,70)$ & 14,33 & 60,02 \\
\hline Alto & $(5,76 ; 8,20,5,30,3,65)$ & 11,91 & 61,08 \\
\hline Medio/bajo & $(1,14 ; 2,60,1,55,1,08)$ & 3,41 & 70,46 \\
\hline
\end{tabular}

El índice del grupo de seguimiento completo, $I_{s c}=14,33$, refleja un resultado satisfactorio y su fase, $F_{s c}=60,02$, un equilibrio casi perfecto en los resultados del aprendizaje dado por los resultados de las diferentes componentes de la evaluación continua.

Si se toma la totalidad de los 340 alumnos, se obtiene un indice $I=11,12$, superior al indice $I=10$ que se obtendría con un simple 5 en cada componente de la evaluación, y una fase $F=61,05$, que manifiesta un aprendizaje suficientemente equilibrado. Para hacer un seguimiento en el tiempo que permita comparar los resultados del curso próximo con los de este año, se propone un índice de evolución $l_{\mathrm{ev}}$ igual al módulo del cuaternión $q_{\mathrm{ev}}$ definido como:

$$
q_{\mathrm{ev}}=\left(\frac{q-q_{0}}{q_{0}}\right) \cdot \frac{100}{4}
$$

donde $q$ es el resultado del curso siguiente y $q_{0}$ el del curso anterior. Debe recordarse que el cuaternión diferencia se obtiene restando las matrices isomorfas de ambos cuaterniones y que el cociente se forma multiplicando por la matriz inversa del cuaternión divisor. También la fase de $q_{\mathrm{ev}}$ indicará si el equilibrio se mantiene o evoluciona, con lo que podrá analizarse la calidad de las evaluaciones en los diferentes componentes.

\section{Conclusiones}

Los resultados globales obtenidos se consideran satisfactorios, tratándose de una primera experiencia. Debe continuarse con la aplicación de la metodología en cursos siguientes.

Se propone valorar el resultado mediante un índice I que englobe los resultados obtenidos en las diferentes componentes de la evaluación que son afectadas por el aprendizaje derivado de la metodología descrita. Es posible valorar un índice $l_{\text {ev }}$ que represente la evolución en el tiempo de los resultados obtenidos.

Existen posibilidades de mejora tales como las que se describen a continuación: El seguimiento del proceso por parte del alumno debe ser completo. No basta con un entrenamiento global para lograr un nivel alto de razonamiento, porque cada tema tiene ideas básicas que deben ser analizadas por el alumno. No deben, por tanto, dejarse cuestionarios de algunos temas sin trabajar. Es importante motivar a los alumnos para lograr superar el actual 49,7\% de seguimiento completo.

Deben crearse cuestiones numéricas en aquellos cuestionarios que actualmente carecen de ellas, para que en todo cuestionario exista al menos una pregunta numérica que obligue al alumno a apoyarse en un cálculo auxiliar, con lo que mejorará la capacidad de cálculo, básica para la resolu- 
ción de problemas y por otro lado disminuye la probabilidad de responder cuestionarios sin el suficiente análisis, buscando puntuaciones altas al azar. De esta forma se aumentará el número de cuestiones a responder en cada cuestionario, lo que aumentará el efecto anterior buscado.
Las mejores cuestiones redactadas por los alumnos en su intento de obtener la calificación de excelencia pueden ser incorporadas a la base de cuestiones, indicando el alumno autor, si este lo autoriza, lo que servirá de estímulo al menos para los mejores alumnos.

\section{BIBLIOGRAFÍA}

Amer, A. (2006): "Reflections on Bloom's revised taxonomy", Electronic Journal of Research in Educational Psychology, vol. 4, pp. 213-230.

European Comission (1999): The Bologna declaration, Communication.

Fernández, C.; Diez, D.; Zarraonandía, T. y Torres, J. (2011): "A student-centered introductory programming course: the cost of applying Bologna principles to compute engineering education", International Journal of Engineering Education, vol. 27, pp. 14-23.

Gurlebeck, K. y Sprossig, W. (1997): Quaternionic and Clifford calculus for physicists and engineers, John Wiley et Sons, Chichester.
Kurt, A. O.; Kubat, C. y Oztemel, E. (2006): "Web-based virtual testing and learning in material science and engineering", International Journal of Engineering Education, vol. 22, pp. 986-992.

Neri, L.; Noguez, J. y Robledo-Rella, V. (2010): "Improving problem-solving skills using adaptive on-line training and learning environments", International Journal of Engineering Education, vol. 26, pp. 1316-1326.

Stefanovic, M.; Matijevic, M.; Cvijetkovic, V. y Simic, V. (2010): "Web-based laboratory for engineering education", Computer Applications in Engineering Education, vol. 18, pp. 526-536. 